Article

\title{
Fluctuating Charge Order: A Universal Phenomenon in Unconventional Superconductivity?
}

\author{
Erminald Bertel * and Alexander Menzel \\ Institute of Physical Chemistry, University of Innsbruck, A-6020 Innsbruck, Austria; \\ alexander.menzel@uibk.ac.at \\ * Correspondence: erminald.bertel@uibk.ac.at; Tel.: +43-512-507-58000 \\ Academic Editor: Sergey Borisenko \\ Received: 6 May 2016; Accepted: 31 May 2016; Published: 10 June 2016
}

\begin{abstract}
Unconventional superconductors are characterized by various competing ordering phenomena in the normal state, such as antiferromagnetism, charge order, orbital order or nematicity. According to a widespread view, antiferromagnetic fluctuations are the dominant ordering phenomenon in cuprates and $\mathrm{Fe}$ based superconductors and are responsible for electron pairing. In contrast, charge order is believed to be subdominant and compete with superconductivity. Here, we argue that fluctuating charge order in the $(0, \pi)$ direction is a feature shared by the cuprates and the Fe based superconductors alike. Recent data and theoretical models suggest that superconductivity is brought about by charge order excitations independently from spin fluctuations. Thus, quantum fluctuations of charge order may provide an alternative to spin fluctuations as a mechanism of electron pairing in unconventional superconductors.
\end{abstract}

Keywords: iron-based superconductors; cuprates; charge density wave; charge order; spin order; fluctuations; phase transition; quantum critical point

\section{Introduction}

Superconductivity arises from the interplay of several mechanisms. First, there is the exchange of bosonic excitations, which gives rise to an indirect electron-electron attraction competing with the Coulomb repulsion. In classical BCS superconductivity, the bosonic excitations are phonons. Basically, an electron with momentum $\mathbf{k}$ scatters into a state $\mathbf{k}-\mathbf{q}$ creating a phonon with energy $\hbar \omega_{q}$. The phonon is absorbed by another electron, whereupon it changes its momentum from $\mathbf{k}^{\prime}$ to $\mathbf{k}^{\prime}+\mathbf{q}$ (see Figure 1). The corresponding interaction is expressed as [1]:

$$
\left\langle i\left|H_{\text {int }}\right| f\right\rangle=\frac{1}{2} \sum_{h}\left\langle f\left|H_{e-p h}\right| h\right\rangle\left\langle h\left|H_{e-p h}\right| i\right\rangle\left(\frac{1}{E_{i}-E_{h}}+\frac{1}{E_{f}-E_{h}}\right)
$$

here, the initial state $|i\rangle=\left|\mathbf{k}, \mathbf{k}^{\prime} ; 0\right\rangle$ is composed of the electron wave functions before scattering and phonon emission. The final state $|f\rangle=\left|\mathbf{k}-\mathbf{q}, \mathbf{k}^{\prime}+\mathbf{q} ; 0\right\rangle$ contains the scattered electron wave functions after scattering and phonon absorption. The intermediate, virtually excited states $|h\rangle=\left|\mathbf{k}-\mathbf{q}, \mathbf{k}^{\prime} ; \hbar \omega_{q}\right\rangle$ with energy $E_{h}=E_{k-q}+E_{k^{\prime}}+\hbar \omega_{q}$ correspond to the excitation of a phonon by one of the electrons. The perturbation operator $H_{e-p h}$ is expressed as:

$$
H_{e-p h}=A_{q} e^{-i \mathbf{q r}} a_{q}^{\dagger}+A_{q}^{*} e^{i \mathbf{q r}} a_{q}
$$

with $a_{q}^{\dagger}$ and $a_{q}$ being the phonon creation and annihilation operators, respectively. $A_{q}$ gauges the strength of the electron-phonon interaction. In order to qualitatively appreciate the meaning of 
Equation (1.1), we assume for simplicity that the scattering amplitude is the same for $\left\langle f\left|H_{e-p h}\right| h\right\rangle$ and $\left\langle i\left|H_{e-p h}\right| h\right\rangle$, and is constant. This yields

$$
\left\langle i\left|H_{\text {int }}\right| f\right\rangle=\frac{1}{2}|M|^{2} \sum_{h}\left(\frac{1}{E_{i}-E_{h}}+\frac{1}{E_{f}-E_{h}}\right)
$$

The summation is subject to the constraint that the electrons can only be scattered into unoccupied states. Note that the virtual states $|h\rangle$ are short-lived and hence need not obey energy conservation. Since $E_{h}$ includes the phonon energy, $\hbar \omega_{q}$, resonance occurs for electron energies $E_{h}^{e^{-}}=E_{i}-\hbar \omega_{q}$ in the virtual state $\left(E_{i} \approx 2 \varepsilon_{k_{F}}\right)$. With hardly any empty states available below $E_{i}-\hbar \omega_{q}$, it is clear that the matrix element Equation (1.3) is negative, i.e., leads to an attractive interaction (see Figure 2).
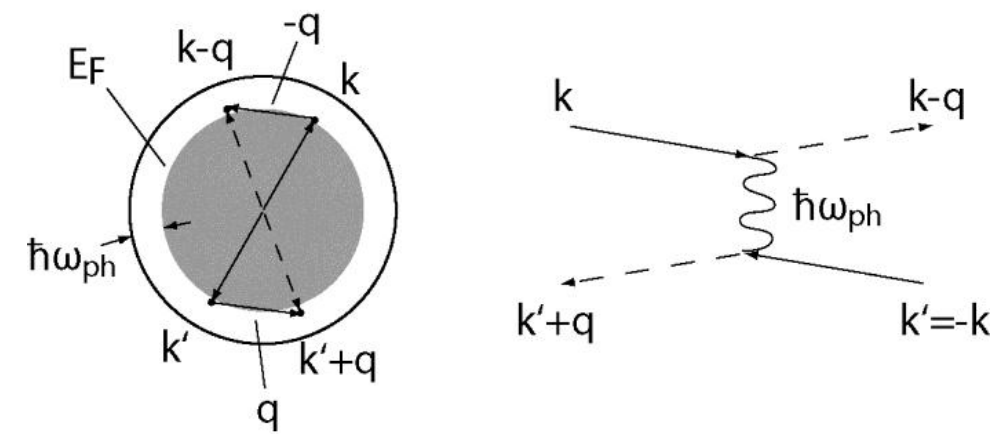

Figure 1. Pairing interaction between electrons at the Fermi level via exchange of phonons $\hbar \omega_{q}$. The diagram refers to one term in the sum Equation (1.1). The total momentum of the electron pair is assumed to be zero (this maximizes the phase space for the summation Equation (1.1)). Only electrons within a shell of width $\hbar \omega_{q}$ around $E_{\mathrm{F}}$ can participate in the pairing interaction.

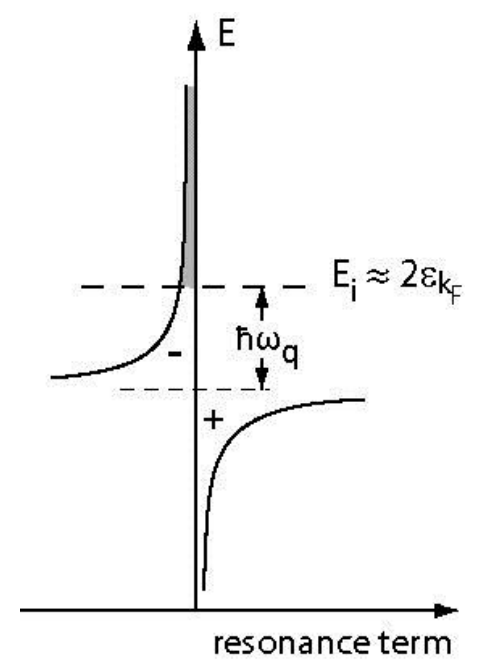

Figure 2. Electron energy diagram for the evaluation of matrix element Equation (1.3). Here, the width of the Fermi distribution is assumed to be negligible in comparison to the phonon energy $\hbar \omega_{q}$. Hence, $E_{i}=E_{f} \approx 2 \varepsilon_{k_{F}}$. The configuration space available for the virtual states is shown as the shaded area.

The attractive interaction Equation (1.3) is a bare interaction competing with the Coulomb repulsion. Whether it can actually give rise to a sufficient Cooper pair density and a superconducting phase transition depends on the response of the other electrons in the Fermi sea. The next step, therefore, is to calculate the Cooper pair susceptibility $\chi_{\text {pair }}(q=0, \omega)$. As mentioned, the choice $q=0$ yields the largest interaction Equation (1.1), and, therefore, in the lowest energy state, the total 
momentum of the pairs is zero, i.e., electron pairs with momentum $k$ and $-k$ are formed. One obtains [2]

$$
\chi_{\text {pair }}(0, \omega)=\frac{\chi_{\text {pair }}^{0}(0, \omega)}{1+U \chi_{\text {pair }}^{0}(0, \omega)}
$$

with

$$
\chi_{\text {pair }}^{0}(0, \omega)=\frac{1}{\Omega} \sum_{k} \frac{f\left(\xi_{k}\right)-f\left(-\xi_{-k}\right)}{\omega-\xi_{k}-\xi_{-k}+i \delta}
$$

$\Omega$ being the volume of the system and $f\left(\xi_{k}\right)$ the Fermi factor at the energy $\xi_{k}=\varepsilon_{k}-E_{F}$. Equation (1.4) signals a phase transition once the denominator goes to zero. Since $\chi_{\text {pair }}^{0}(0, \omega)$ is positive, this may be the case for attractive interactions $U(U<0)$ such as Equation (1.3).

The exchange of phonons, however, is not the only possible source for attractive electron interactions. As mentioned above, other bosonic excitations could serve a similar purpose. In particular, there is a general consensus that paramagnon excitations are likely candidates in the case of cuprates and also of the iron-based unconventional superconductors. A third possibility arises in the presence of (fluctuating) charge density waves (CDW). The complex CDW order parameter is characterized by the amplitude and the phase of the CDW modulation. The normal modes of these two degrees of freedom are amplitudon and phason [3] (see Figure 3). The dispersion of the normal modes resembles the dispersion of optical and acustic phonons, respectively. For an incommensurate CDW, a uniform phase shift of the charge modulation relative to the ionic lattice does not change the energy, thus the phason mode dispersion starts at $\omega_{\text {phason }}=0$ for $q=0$. In contrast, the amplitudon has a finite energy even at $q=0$. Note that the phason involves a shift of the electronic charge with respect to the ionic background and therefore is associated with an optically excitable dipole moment, while the amplitudon changes the polarizability and is detectable in Raman spectroscopy.

A static antiferromagnetic or CDW order is detrimental to superconductivity because it preempts the carrier reservoir available for the pairing mechanism sketched above. In the cuprates, for instance, doping is required to suppress the static antiferromagnetic order parameter, thus allowing the superconducting phase to evolve. Similarly, superconductivity and static CDW order are mutually exclusive, for instance, in $\mathrm{La}_{1 .} \mathrm{Sr}_{0.1} \mathrm{CuO}_{4}$ [3]. Therefore, the unconventional mechanisms involving magnetic and charge order excitations require tuning into a fluctuating regime. This naturally explains the affinity of the superconducting phase transition to a quantum critical point. In addition, the static susceptibility is directly linked to the fluctuations of the order parameter $m$ via the fluctuation-dissipation theorem:

$$
\chi(\omega=0, T)=\frac{1}{k_{B} T}\left(\left\langle m^{2}\right\rangle-\langle m\rangle^{2}\right)
$$

In other words, the presence of a fluctuating order parameter amplifies the response of the material to an external potential. A large $\chi_{C D W}$ leads to a strong coupling between electrons and the charge order excitations shown in Figure 3. 

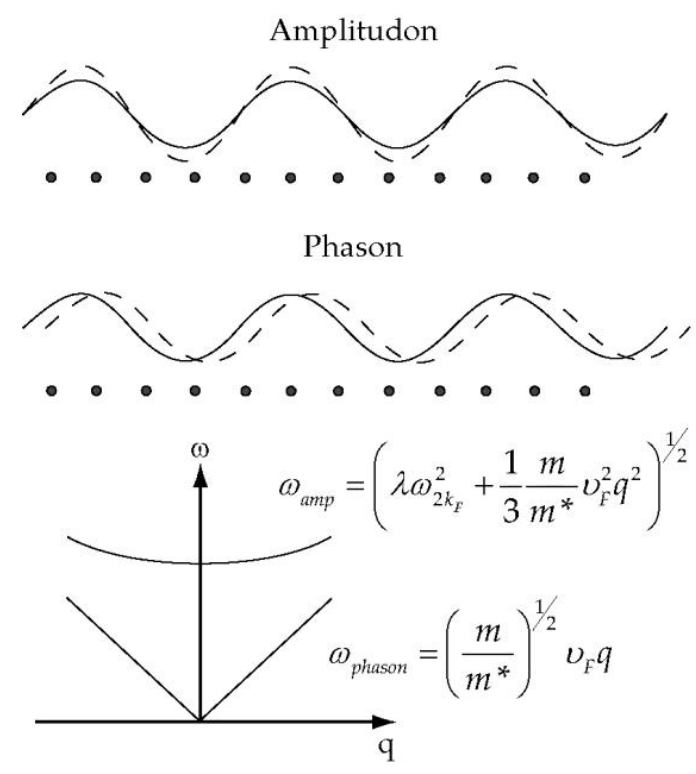

Figure 3. Schematic representation of amplitudon and phason modes in an incommensurate charge ordered state and the corresponding dispersion relation [4].

Fluctuating CDW order parameters have been detected, for example, in cuprates [3] and are also borne out by theoretical models of the iron based superconductors [5]. It is not entirely clear whether fluctuating charge order competes with superconductivity or favors it [3,6-11], but we will come back to this point further below. Direct evidence of fluctuating CDW order in Fe based superconductors is scarce and theoretical models are based on simplified model band structures. The problem stems in part from a fundamental difficulty of probing the relevant order parameters in superconductors. Angle-resolved photoemission spectroscopy (ARPES), scanning tunneling microscopy (STM) and scanning tunneling spectroscopy (STS) belong to the most powerful techniques applied to explore the nature of the order parameters competing in the parameter space of unconventional superconductivity. However, these techniques are very surface sensitive and therefore subject to some constraints. Surfaces may exhibit carrier densities different from the bulk, they can be geometrically and electronically reconstructed, and the order parameter prevalent in the bulk may deviate at the surface. Hence, it is not guaranteed that the results of surface sensitive methods mirror the actual state of affairs in the bulk. In the following, we choose a particular example to illustrate this point. We discuss how, on the one hand, the presence of the surface may interfere with probing the order parameter, while, on the other hand, may indirectly reveal important clues about the bulk order parameter. Furthermore, the surface is accessible to modifications on the atomic scale, thus opening alternative ways of obtaining information.

\section{The 122 Iron Pnictides}

\subsection{The 122 Cleavage Surfaces}

Recent investigations of the surface structure of 122 Fe pnictides sparked a controversy about the origin of the surface structures observed by STM and Low Energy Electron Diffraction (LEED). In order to appreciate the problem, it is useful to rehearse the structural details of the 122 compounds as illustrated in Figure 4. It shows the real space crystal structure, the surface structure for different surface ordered phases after cleavage and the surface Brillouin zone of a hypothetical $(1 \times 1)$ surface phase. Common structural elements are Fe planes sandwiched between As atoms and $A$-planes $(A=\mathrm{Ca}, \mathrm{Sr}, \mathrm{Ba})$ separating the As-Fe-As sandwiches. The Fe atoms are tetrahedrally coordinated to the As atoms, and, in the undoped parent compounds, they exhibit row-to-row antiferromagnetism as indicated in Figure 4c. 
The crystals cleave in the A-plane and different groups disagreed over whether the A atoms are evenly distributed over both sides yielding nonpolar surfaces or whether the A atoms remain essentially on one side of the cleavage plane yielding polar surfaces. STM studies showed, depending on the cleavage procedure, $(1 \times 2), \mathrm{c}(2 \times 2)$ or disordered surface structures. The ordered structures seemed to favor a half-monolayer coverage expected for nonpolar surfaces [12-15], but were alternatively also interpreted as being due to a structural reconstruction in the FeAs layer [16,17] or attributed to the local spin order [18]. The controversy is reviewed in [19]. It seems that the controversy settled more or less in favor of nonpolar surfaces with half-monolayer coverage [19-21].

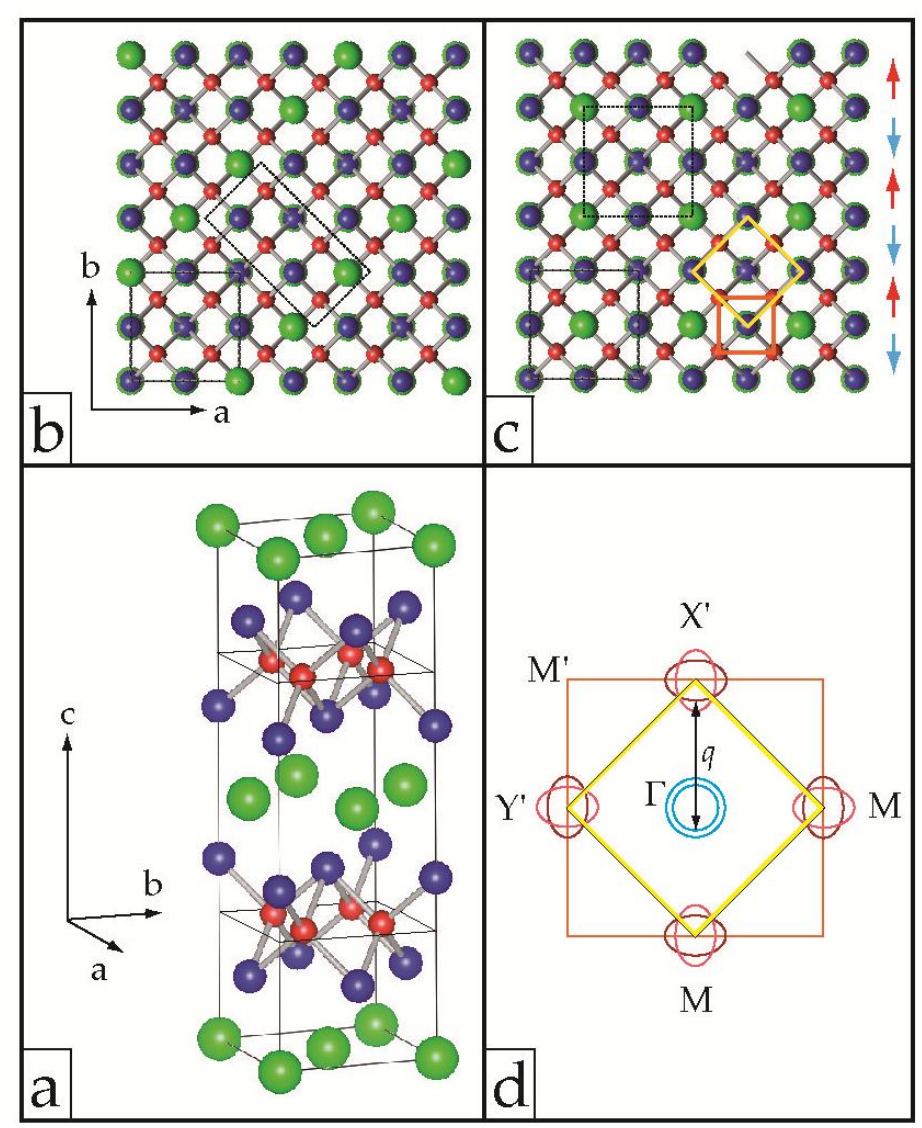

Figure 4. (a) crystal structure of the 122 Fe pnictides; red spheres: Fe, blue spheres: As, green spheres: $\mathrm{Ca}, \mathrm{Sr}$, or $\mathrm{Ba} ;(\mathbf{b})(2 \times 1)$ surface structure prevailing after cleavage at low temperature; black square: basal plane of the unit cell shown in $(\mathbf{a})$, black dotted rectangle: $(2 \times 1)$ surface unit cell seen in LEED and STM. For clarity, it has been offset relative to the atom positions; $(\mathbf{c}) \mathrm{c}(2 \times 2)$ structure observed in LEED and STM after cleavage at room temperature; black dotted square: $(\sqrt{ } 2 \times \sqrt{ } 2) \mathrm{R} 45^{\circ}$ primitive surface unit cell; orange square: conventional unit cell (defined with regard to a simple, square iron lattice) including one Fe atom; yellow square: actual two-Fe unit cell; and (d) unfolded and downfolded surface Brillouin zones (SBZ) resulting from the orange and yellow unit cells, respectively, shown in (c). Principal points of the unfolded SBZ are primed; blue circles: Hole Fermi surfaces around the $\Gamma$ point; dark red ellipses: electron Fermi surfaces; light red ellipses: downfolded electron Fermi surfaces. The spin density wave (SDW) vector $q$ connects the $\Gamma$ hole Fermi surface and the $M$ electron Fermi surface (downfolded BZ).

\subsection{The Order-Order Phase Transition}

Accepting that the surface structure of the 122 compounds is caused by a half-monolayer coverage of A atoms, one faces another puzzle: Low-temperature cleavage results in a somewhat defective $(2 \times 1)$ order, while cleavage at room temperature yields a $\mathrm{c}(2 \times 2)$ structure (note that the elementary 
unit cell of the latter structure is $(\sqrt{ } 2 \times \sqrt{ } 2) R 45^{\circ}$; see Figure 4). A temperature induced phase transition between two ordered adsorbate structures is a rare case, since it requires an entropy gain in the high-temperature phase. If the high- $T$ phase is ordered as well, configurational entropy cannot significantly contribute (moreover, in the present case, the high- $T$ phase seems to be better ordered than the low- $T$ phase). Vibrational entropy can make a difference [22], but the local adsorption site of the $\mathrm{A}$ atoms is the same on the 122 cleavage surfaces in both the low- $T$ and high- $T$ phases, and therefore the change in vibrational entropy is expected to be marginal. The remaining possibility is an entropy contribution from the substrate. The latter alternative is supported by the notion that most $T$-induced order-order transitions on surfaces reported so far refer to hydrogen adsorption systems. $\mathrm{H}$ is notorious for inducing substrate reconstructions, and, in most cases, the order-order transition can be traced to the lifting of these substrate reconstructions in the high- $T$ phase.

A similar type of substrate induced order-order transition in the adsorbate layer has been explored on the Pt(110) surface with a coverage of 0.5 monolayers (ML) of $\mathrm{Br}[20,23,24]$. Pt exhibits a strong Kohn anomaly in its phonon dispersion, signaling a coupling of the phonons to electronic excitations with finite wave vector $q$ at $E_{\mathrm{F}}$. On the strongly anisotropic (110) surface, this results in a tendency to develop a surface CDW [25]. Saddle-points in the surface electronic structure [26-28] at $E_{\mathrm{F}}$ favor a CDW phase by providing a large joint density of states. However, since the corresponding $q$ vector is incommensurate, the CDW is not stable on the clean $\mathrm{Pt}(110)$ surface, at least not above $50 \mathrm{~K}$. In the presence of 0.5 to $0.66 \mathrm{ML}$ of $\mathrm{Br}$, however, CDW fluctuations are observed. At a coverage of precisely $0.5 \mathrm{ML}$, the well ordered room temperature $\mathrm{c}(2 \times 2)-\mathrm{Br} / \mathrm{Pt}(110)$ structure shows a phase transition to a low- $T(2 \times 1)$ structure at around $50 \mathrm{~K}[23,29]$. Fluctuating $(2 \times 1)$ domains can be imaged by STM in this case, since the fluctuations are rather sluggish at $50 \mathrm{~K}$ [29] (see Figure 5a). For slightly higher coverages, CDW fluctuations with a threefold periodicity emerge. At room temperature, these fluctuations can only be resolved in the presence of defects, which locally pin fluctuating domains (Figure 5b). On unperturbed terraces, the only sign of the fluctuations is the loss of atomic resolution, since the STM yields a time-averaged image. Figure $5 \mathrm{c}$ is an artist's rendering of fluctuating CDW domains as detected in ultrafast pump-probe spectroscopy on $\mathrm{La}_{1.84} \mathrm{Sr}_{0.16} \mathrm{CuO}_{4}$ [3]. Of course, correlation lengths cannot be determined from an experiment in the time domain but are accessible in diffraction experiments (see e.g., [8]). The similarity with the structures imaged on the $\mathrm{Br} / \mathrm{Pt}(110)$ surface is evident.

Figure $5 \mathrm{~b}$ illustrates yet another aspect of fluctuating charge order: While defects cause a local phase pinning of CDW domains, in general, this does not result in a global charge ordering. Figure $5 \mathrm{~b}$ shows a coverage of $0.55 \mathrm{ML}$ of $\mathrm{Br}$ atoms on the $\mathrm{Pt}(110)$ surface. However, in the space between the pinned domains, only a $(1 \times 1)$ contrast is visible. This is due to the fluctuation driven mobility of the $\mathrm{Br}$ atoms, which, in the time averaged STM image, visit every adsorption site with equal probability [25]. Analogously, one could envision that defect pinning of the charge fluctuations in superconductors does not necessarily lead to a global static charge ordering. For random defect distribution, one should expect a cross-over from an inherent temperature dependent CDW correlation length at low defect density to an extrinsic correlation length $\propto \frac{1}{d}$, where $d$ is the average distance between defects.

The interaction of the adsorbate with the fluctuating CDW in the substrate can be rationalized in a simple one-dimensional model. The model contains a Landau expansion of the free energy in terms of the order parameter $m$ to describe the substrate CDW order, an Ising type modeling of interadsorbate repulsion with interaction parameter, $J$, and a coupling term with coupling strength, $g(<0)$, capturing the modulation of the adsorbate-substrate bond strength by the CDW:

$$
F=\sum_{i} J s_{i} s_{i+1}+\sum_{i} g m\left(s_{i}+s_{i+1}\right)+\alpha(T) m^{2}+\lambda m^{4}
$$

here, $s_{i}$ is the occupation number of site $i$ ( $\pm \frac{1}{2}$ for occupied and unoccupied sites, respectively), and $\alpha(T)$ is the usual $T$-dependent Landau coefficient changing sign at the critical temperature. The model is illustrated in Figure 6 and discussed in more detail in [23]. Basically, in the high- $T$ phase, the CDW 
order parameter is zero and the adsorbate structure is determined by the interadsorbate repulsion. For half-monolayer coverage, this results in a $c(2 \times 2)$ structure. In the low- $T$ phase, $m \neq 0$, and, therefore, the coupling term lowers the energy, if neighboring sites are occupied, as is the case in the $(2 \times 1)$ but not in the $c(2 \times 2)$ structure. For sufficiently strong coupling, this energy gain surpasses the interadsorbate repulsion, and the surface adopts a $(2 \times 1)$ structure. Note that the coupling term plays the role of an external field in the Landau expansion of the free energy, so it assists in driving the order parameter to a finite value $m>0$. In other words, the adsorbate elicits the CDW order in a kind of bootstrap mechanism.
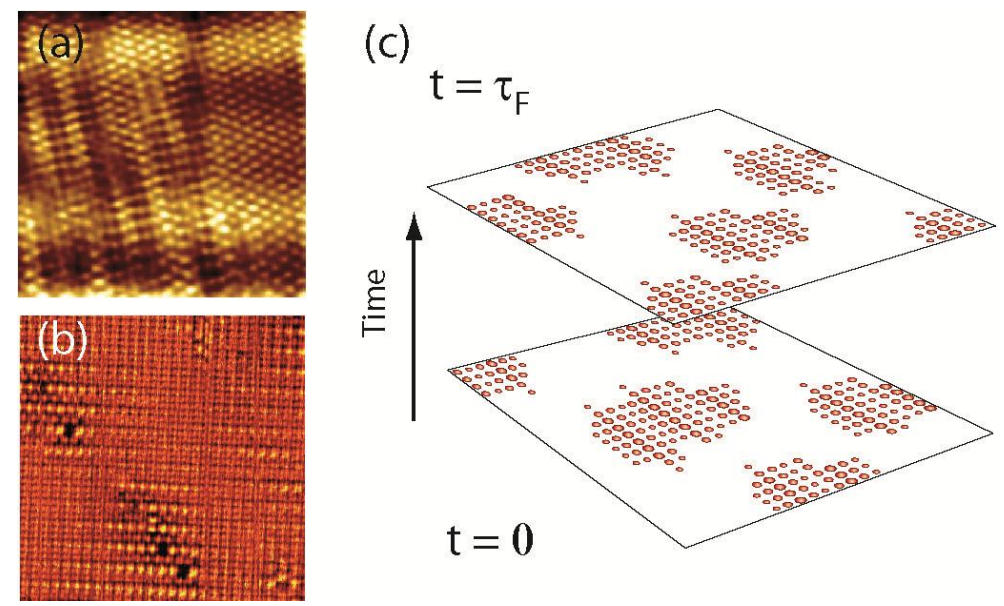

Figure 5. (a) STM image of fluctuating $(2 \times 1)$ domains in a $\mathrm{c}(2 \times 2)$ adsorbate structure $(0.5 \mathrm{ML}$ $\mathrm{Br} / \mathrm{Pt}(110))$ recorded at $\mathrm{T}=50 \mathrm{~K}$ [29]. Imaging of the time-dependent domains is possible, since the fluctuations are slow at $50 \mathrm{~K}$; (b) STM image of $(3 \times 1)$ domains $(0.55 \mathrm{ML} \mathrm{Br} / \mathrm{Pt}(110))$ recorded at room temperature [25]. Here, imaging of the domains is possible, because they are pinned at defects. At some distance from the defects, a $(1 \times 1)$ contrast is obtained indicating equal occupation probability for every adsorption site in a fluctuating adsorbate layer; and (c) an artist's rendering of fluctuating CDW domains in cuprate superconductors (redrawn after [3]).

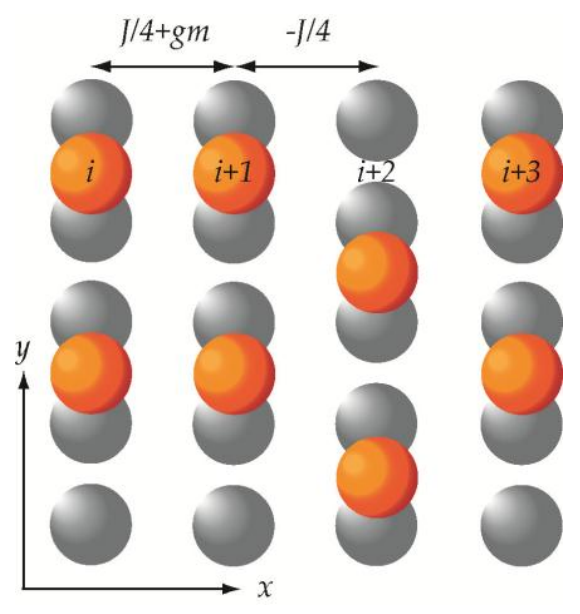

Figure 6. Interactions in the adsorbate layer according to Equation (2.1), shown here for the $\mathrm{Br} / \mathrm{Pt}(110)$ system. The substrate contribution to the free energy $\alpha(T) m^{2}+\lambda m^{4}$ is not included in the Figure. Interactions in the $y$-direction are not explicitly taken into account, since they are the same in $(2 \times 1)$ order shown in the left part and the $\mathrm{c}(2 \times 2)$ order shown in the right part. Note that the coupling constant, $g$, is negative, so that, for sufficiently strong coupling, $(2 \times 1)$ wins over $c(2 \times 2)$ order, provided $m \neq 0$. 
Obviously, such a model could explain the T-dependence of surface phases also in the $122 \mathrm{Fe}$ superconductors. In fact, the surface topology in the coexistence range of $c(2 \times 2)$ and $(2 \times 1)$ is strikingly similar for $\mathrm{Br} / \mathrm{Pt}(110)$ and $A / \mathrm{Fe}_{2} \mathrm{As}_{2}$ (see Figure 3 in [20]). In contrast to $\mathrm{Br}$ on the $\mathrm{Pt}(110)$ surface, however, the mobility of the $A$ atoms on the cleavage surfaces seems to be low. Therefore, the pattern is determined during the cleavage process. At low temperature, the $(2 \times 1)$ order is preferred. Upon heating, the CDW melts and allows the adatoms to switch to the $\mathrm{c}(2 \times 2)$ order. However, the transition is irreversible, since cooling again to the critical temperature suppresses the mobility of the adatoms. Thus, the low-T structure seems to be metastable [19], but it is actually the groundstate at low $T$. It cannot be reached from the high- $T$ phase due to kinetic hindrance and can only be accessed directly in the cleavage process.

Furthermore, it is to be stressed that the situation in the $\mathrm{AFe}_{2} \mathrm{As}_{2}$ bulk is different: here, the $\mathrm{Fe}_{2} \mathrm{As}_{2}$ layers are separated by complete monolayers of $\mathrm{A}$ atoms, hence the bootstrap mechanism Equation (2.1) cannot operate. Consequently, CDW fluctuations prevail and no static CDW order is established.

\subsection{The Fermi Topology of 122 Fe Pnictides}

The parallels drawn above between $\mathrm{Br} / \mathrm{Pt}(110)$ and $\mathrm{AFe}_{2} \mathrm{As}_{2}$ are alluring, but the postulated CDW wave vector does not match the Fermi surface (FS) topology assumed in the simplified band structure models for $\mathrm{AFe}_{2} \mathrm{As}_{2}$. The fluctuating CDW is not in the direction of the SDW, instead it is offset by $45^{\circ}$ (see Figure 4), as pointed out by us previously [20]. A commensurate CDW which doubles the periodicity yielding a $(2 \times 1)$ unit cell would correspond to a $(0, \pi)$ or $(\pi, 0)$ wave vector with respect to the downfolded SBZ. Since the CDW order parameter fluctuates, the wave vector is apparently (slightly) incommensurate, but in any case is rotated by $45^{\circ}$ with respect to the $(\pi, \pi)$ SDW wave vector.

The apparent discrepancy with the FS topology, however, may be an artifact of the simplified model band structure. Recent ARPES data hint towards a more complicated FS topology [30-37], at least at the M point of the downfolded SBZ. The related ARPES structures appear with different names in literature, such as propeller structures [32,36] or droplet FS [34]. The origin of these structures is subject to controversial interpretations. Originally, there appeared to be consensus that the complex FS topology results from $(\pi, \pi)$ electronic ordering $[33,37]$. More recently, alternative explanations have been brought forward for the appearance of flat bands at $E_{\mathrm{F}}$ that are not predicted in DFT calculations. They are based on the observation of Lifshitz transitions $[32,38]$ as a function of doping and involve orbital dependent renormalization, Hund's rule coupling and other strong correlation effects. Regardless of the origin, $(\pi, \pi)$ backfolding or correlation effects, the ARPES data imply a proximity of the Fermi energy to singular points (van Hove singularities, saddle points, etc.) in the band structure.

$(\pi, \pi)$ backfolding will of course affect the $\Gamma$ and $M$ points on equal footing. Kondo et al. [33] found a pronounced incommensurate FS nesting at the central hole pocket for $\mathrm{BaFe}_{2} \mathrm{As}_{2}$ and $\mathrm{CaFe}_{2} \mathrm{As}_{2}$ not expected on the basis of DFT calculations. Consistent with the considerations above, they attributed this deformation of the $\Gamma$ point FS to a $(\pi, \pi)$ backfolding from the M point. The extended FS nesting with nesting vectors $(0.15 \pi, 0.15 \pi)$ and $(0.3 \pi, 0.3 \pi)$ was proposed to be a precursor for CDW correlations. According to this proposal, however, the incipient charge order would have a different wavelength but the same direction as the AF modulation.

Whatever the origin of the flat bands at $E_{\mathrm{F}}$ might be, there are regions of strongly enhanced DOS close to $E_{\mathrm{F}}$, particularly in the vicinity of the electron pockets at the $\mathrm{M}$ points. Scattering between these hot spots ("interpocket scattering") can cause electronic instabilities by themselves, e.g., induce CDW fluctuations. Figure 7 shows schematically an incommensurate $q$-vector in $(0, \pi)$ direction connecting areas of high DOS at $E_{\mathrm{F}}$. Note that extended FS nesting is not a unique prerequisite for CDW order to appear $[26,39,40]$. A more rigorous approach is the calculation of the susceptibility $\chi(q)$ [39-41]. This, of course, is anything but trivial in view of the differences mentioned above between the DFT band structure and the ARPES results. 


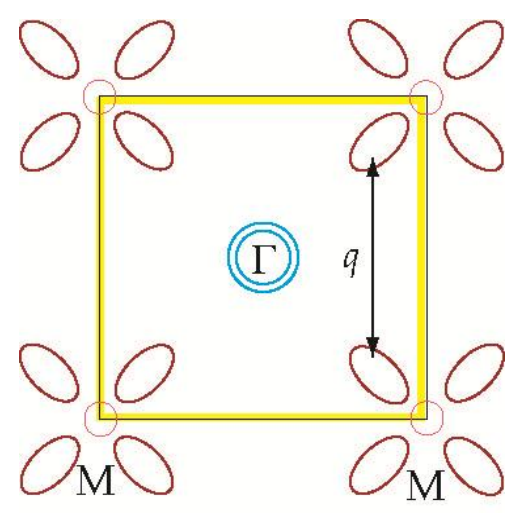

Figure 7. The flowerlike Fermi surfaces at $\mathrm{M}$ enclose regions of enhanced DOS close to $E_{\mathrm{F}}$. Scattering between those "hot spots" may induce an (incommensurate, fluctuating) CDW order.

Additional evidence for the importance of this interpocket scattering in the $(0, \pi)$ direction stems from recent ARUPS and STM results on $\mathrm{K}$ doped (i.e., electron doped) FeSe superconducting films. They have revealed the existence of two superconducting domes in the $T_{\mathrm{c}}$ versus doping phase diagram $[42,43]$. This suggests two different pairing mechanisms to be at work. For low electron doping, the spin fluctuation mechanism mediating $s_{ \pm}$pairing seems plausible, but at higher doping, the hole pocket at $\Gamma$ disappears together with the superconductivity. Upon even further doping, a second superconducting dome appears and scattering between the $\mathrm{M}$ point electron pockets apparently plays an essential role in the related pairing mechanism.

Summarizing, the $(2 \times 1)$ order revealed on the surface of the 122 compounds after low-temperature cleavage is interpreted here as originating from charge order trapped by the half-monolayer of $A$ atoms at the cleavage plane. In the bulk, the charge order is slightly incommensurate and fluctuates. Most significantly, the direction of the charge order wave vector is axial with respect to the downfolded SBZ, while the spin order is diagonal. For other Fe based superconductors, the preferred periodicity may be different, but the appearance of strong scattering in the $(0, \pi)$ direction from $\mathrm{M}$ to $\mathrm{M}$ and its relation to the pairing mechanism is apparently a rather general phenomenon.

\section{Charge Order in Cuprates and Other Unconventional Superconductors}

Recent experiments in cuprates have revealed ample evidence for incommensurate charge modulations with wavevectors oriented along the crystalline axes, i.e., at $45^{\circ}$ off the SDW vector as well [8,44-54]. A theoretical investigation examining a multiorbital model of cuprates finds support for such an axial charge order [46] in contrast to previous calculations, which reported wave vectors along the Brillouin zone diagonal. According to this study, the ordering of the local moments leads to a Fermi surface reconstruction, which, in turn, gives rise to charge order in $(0, \pi)$ resp. $(\pi, 0)$ direction. As an alternative to unidirectional CDW domains, checkerboard charge ordering has been proposed in some cases, but at least for YBCO [49,55,56], superposition of two unidirectionally charge ordered domains seems to reflect the true structure.

This is a striking parallel to the present scenario proposed for the Fe based superconductors. Already Castellani et al. [6] discussed for the cuprates an association of the superconducting dome with the quantum critical point of an incommensurate charge density wave. More specifically, Wang and Chubukov [57] recently investigated the interplay between superconductivity and axial CDW order in the cuprates. They propose a phase diagram with two separate superconducting domes, one above the quantum critical point of the antiferromagnetic order with superconductivity mediated by spin fluctuations and one above the quantum critical point of the charge order with superconductivity mediated by the CDW fluctuations. Whether the two superconducting domes actually appear separated by an intervening phase in the phase diagram depends, of course, on the position of 
the two quantum critical points. In any case, the similarity with the phase diagram revealed by the electron doping experiments via $\mathrm{K}$ evaporation onto FeSe films is striking.

Further evidence for a critical role of charge density fluctuations stems from a study of structurally related $\mathrm{Pt}$ based superconductors. Soshi and Motoharu [58] suggest that superconductivity in $\mathrm{SrPt}_{2} \mathrm{As}_{2}$ is enhanced, not suppressed, by the presence of charge ordering. A previous study of $2 \mathrm{H}-\mathrm{NbSe}_{2}$ by Kiss et al. [59] also demonstrated that charge order can boost superconductivity, rather than competing with it. Finally, one should note that fluctuating charge order close to a quantum critical point is also observed in organic superconductors [60]. Thus, a universal mechanism involving charge order fluctuations seems to be at work in several classes of unconventional superconductors. For the cuprates and Fe based superconductors in particular, fluctuating charge order in $(0, \pi)$ and $(\pi, 0)$ direction-as opposed to spin fluctuations in the $(\pi, \pi)$ direction-seem to offer an alternative pair bonding mechanism via exchange of charge order excitations.

\section{Experimental Considerations}

As mentioned above, the use of surface sensitive techniques imposes certain restrictions on the extraction of bulk information. For instance, in the case of the 122 iron arsenide superconductors, the details of the cleavage process fix different boundary conditions for the CDW fluctuations. If carried out at low temperature, a $(2 \times 1)$ order is created by trapping (at least locally) a static CDW order. At room temperature, cleavage results in a $\mathrm{c}(2 \times 2)$ or a disordered surface, which suppresses charge order. Figure 5 illustrates how even single defects pin otherwise fluctuating CDW domains. Given a higher random defect density, the domains would destructively interfere and thus disappear entirely.

However, surface sensitive measurements also provide a unique opportunity: the surface can be manipulated with atomic precision. This has been demonstrated recently by the success of electron doping via K adsorption on FeSe films [42,43,61]. A similar strategy could be used to switch on and off the CDW fluctuations in the surface region of the $122 \mathrm{Fe}$ compounds. The static $(2 \times 1) \mathrm{CDW}$ order pinned after low- $T$ cleavage by the adsorbate can be destroyed by further evaporation of $A$ atoms onto the surface. As the coverage approaches a full monolayer, similar conditions as in the bulk are attained, disorder is removed, and, therefore, the charge density fluctuations should be restored. While adsorption of additional A atoms changes the surface dipole and may therefore influence the charge density in the topmost layers, variation of the bulk doping level should offer an independent handle to control this effect. A study along this lines should provide some additional insight into the interplay between charge order and superconductivity in $122 \mathrm{Fe}$ based superconductors.

As for the search for other materials exhibiting unconventional superconductivity, the present considerations suggest examining materials close to a quantum critical point, where charge order disappears, either as a consequence of doping, "chemical pressure" or increased coupling between two-dimensional layers. It is apparently not clear yet whether fluctuating charge order alone or only in cooperation with fluctuating spin order can cause superconductivity. On the one hand, the recent finding of two separated superconducting domes hints towards the former possibility. On the other hand, the complex FS topology of the Fe based superconductors that enables the intense interpocket scattering is likely caused by $(\pi, \pi)$ backfolding, and, in that case, would not exist without spin order. Naturally, charge ordered phases are more likely to occur in low-dimensional materials. It would be interesting to study other layered materials with charge order and tune them close to the quantum critical point. Finally, in the compounds discussed so far, charge ordering can occur in two equivalent directions. The investigation of anisotropic materials, where only one domain orientation is possible for the fluctuating charge order, should provide additional insights.

\section{Conclusions}

Spin fluctuations are manifest in a vast majority of high- $T_{\mathrm{c}}$ superconductors. In contrast, the presence and the precise nature of fluctuating charge order is much more disputed. This may in part be due to the fact that surface sensitive methods tend to miss fluctuating charge order, since it is 
suppressed by defects or frozen into static domains by the coupling to surface atoms. The latter effect, however, can also be used to infer the presence of charge order fluctuations in the bulk, as demonstrated here on the example of the $\mathrm{AFe}_{2} \mathrm{As}_{2}$ compounds. The resulting pattern of $\mathrm{CDW}$ fluctuations, with wave vector in most cases being different from the spin ordering, seems to universally apply for iron-based as well as Cuprate superconductors. Together with recent phase diagrams reported for electron doped FeSe and for cuprates, it appears that superconductivity can arise close to a quantum critical point for charge ordering, perhaps even independently of the quantum critical point for spin order. This challenges the widespread view of charge order being detrimental to superconductivity. Instead, bosonic charge order excitations may offer an alternative mechanism for electron pairing.

Acknowledgments: Part of the research has been supported by the Austrian Science Funds. We thank Norbert Memmel for useful discussions.

Author Contributions: Both authors discussed the contents of the manuscript and co-operated in its preparation. Alexander Menzel contributed in particular to section one and Erminald Bertel wrote the paper.

Conflicts of Interest: The authors declare no conflict of interest.

\section{Abbreviations}

The following abbreviations are used in this manuscript:

$\begin{array}{ll}\text { ARPES } & \text { Angle-resolved photoelectron spectroscopy } \\ \text { BCS } & \text { Bardeen-Cooper-Schrieffer } \\ \text { BZ } & \text { Brillouin zone } \\ \text { CDW } & \text { Charge density wave } \\ \text { DFT } & \text { Density functional theory } \\ \text { DOS } & \text { Density of states } \\ \text { FS } & \text { Fermi surface } \\ \text { ML } & \text { Monolayer } \\ \text { SBZ } & \text { Surface Brillouin zone } \\ \text { SDW } & \text { Spin density wave } \\ \text { STM } & \text { Scanning tunneling microscopy } \\ \text { STS } & \text { Scanning tunneling spectroscopy } \\ \text { YBCO } & \text { Yttrium barium cuprate }\end{array}$

\section{References}

1. Grosso, G.; Parravicini, G. Solid State Physics; Elsevier Academic Press: Oxford, UK, 2014.

2. Giamarchi, T. Quantum Physics in One Dimension; Oxford University Press: New York, NY, USA, 2004.

3. Torchinsky, D.H.; Mahmood, F.; Bollinger, A.T.; Božović, I.; Gedik, N. Fluctuating charge-density waves in a cuprate superconductor. Nat. Mater. 2013, 12, 387-391. [CrossRef] [PubMed]

4. Grüner, G. Density Waves in Solids; Perseus Publishing: Cambridge, MA, USA, 1994.

5. Wang, F.; Lee, D.-H. The electron-pairing mechanism of iron-based superconductors. Science 2011, 332, 200-204. [CrossRef] [PubMed]

6. Castellani, C.; di Castro, C.; Grilli, M. Non-fermi-liquid behavior and d-wave superconductivity near the charge-density-wave quantum critical point. Z. Phys. B Condens. Matter 1996, 103, 137-144. [CrossRef]

7. Castellani, C.; di Castro, C.; Grilli, M. Singular quasiparticle scattering in the proximity of charge instabilities. Phys. Rev. Lett. 1995, 75, 4650-4653. [CrossRef] [PubMed]

8. Croft, T.P.; Lester, C.; Senn, M.S.; Bombardi, A.; Hayden, S.M. Charge density wave fluctuations in $\mathrm{La}_{2-x} \mathrm{Sr}_{x} \mathrm{CuO}_{4}$ and their competition with superconductivity. Phys. Rev. B 2014, 89, 224513. [CrossRef]

9. Thampy, V.; Dean, M.P.M.; Christensen, N.B.; Steinke, L.; Islam, Z.; Oda, M.; Ido, M.; Momono, N.; Wilkins, S.B.; Hill, J.P. Rotated stripe order and its competition with superconductivity in $\mathrm{La}_{1.88} \mathrm{Sr}_{0.12} \mathrm{CuO}_{4}$. Phys. Rev. B 2014, 90, 100510. [CrossRef]

10. Noack, R.M.; Scalapino, D.J.; Scalettar, R.T. Charge-density-wave and pairing susceptibilities in a two-dimensional electron-phonon model. Phys. Rev. Lett. 1991, 66, 778-781. [CrossRef] [PubMed]

11. Zhai, H.; Wang, F.; Lee, D.-H. Antiferromagnetically driven electronic correlations in iron pnictides and cuprates. Phys. Rev. B 2009, 80, 064517. [CrossRef] 
12. Yin, Y.; Zech, M.; Williams, T.L.; Wang, X.F.; Wu, G.; Chen, X.H.; Hoffman, J.E. Scanning tunneling spectroscopy and vortex imaging in the iron pnictide superconductor $\mathrm{BaFe}_{1.8} \mathrm{Co}_{0.2} \mathrm{As}_{2}$. Phys. Rev. Lett. 2009, 102, 097002. [CrossRef] [PubMed]

13. Hsieh, D.; Xia, Y.; Wray, L.; Qian, D.; Gomes, K.K.; Yazdani, A.; Chen, G.F.; Luo, J.L.; Wang, N.L.; Hasan, M.Z. Experimental determination of the microscopic origin of magnetism in parent iron pnictides. Available online: https: / / arxiv.org/abs/0812.2289 (acessed on 6 June 2016).

14. Massee, F.; de Jong, S.; Huang, Y.; Kaas, J.; van Heumen, E.; Goedkoop, J.B.; Golden, M.S. Cleavage surfaces of the $\mathrm{BaFe}_{2-x} \mathrm{Co}_{x} \mathrm{As}_{2}$ and $\mathrm{Fe}_{y} \mathrm{Se}_{1-x} \mathrm{Te}_{x}$ superconductors: A combined STM plus LEED study. Phys. Rev. B 2009, 80, 140507. [CrossRef]

15. Zhang, H.; Dai, J.; Zhang, Y.; Qu, D.; Ji, H.; Wu, G.; Wang, X.F.; Chen, X.H.; Wang, B.; Zeng, C.; et al. $\sqrt{ } 2 \times \sqrt{ } 2$ structure and charge inhomogeneity at the surface of superconducting $\mathrm{BaFe}_{2-x} \mathrm{Co}_{x} \mathrm{As}_{2}(x=0-0.32)$. Phys. Rev. B 2010, 81, 104520. [CrossRef]

16. Nascimento, V.B.; Li, A.; Jayasundara, D.R.; Xuan, Y.; O’Neal, J.; Pan, S.; Chien, T.Y.; Hu, B.; He, X.B.; Li, G.; et al. Surface geometric and electronic structures of $\mathrm{BaFe}_{2} \mathrm{As}_{2}(001)$. Phys. Rev. Lett. 2009, 103, 076104. [CrossRef] [PubMed]

17. Niestemski, F.C.; Nascimento, V.B.; Hu, B.; Plummer, E.W.; Gillett, J.; Sebastian, S.; Wang, Z.; Madhavan, V. Unveiling the atomic and electronic structure at the surface of the parent pnictide $\mathrm{SrFe}_{2} \mathrm{As}_{2}$. Available online: http:/ / arxiv.org/abs/0906.2761 (acessed on 6 June 2016).

18. Li, G.; He, X.; Li, A.; Pan, S.H.; Zhang, J.; Jin, R.; Sefat, A.S.; McGuire, M.A.; Mandrus, D.G.; Sales, B.C.; et al. $\mathrm{BaFe}_{2} \mathrm{As}_{2}$ surface domains and domain walls: Mirroring the bulk spin structure. Available online: http:/ / arxiv.org/abs/1006.5907 (acessed on 6 June 2016).

19. Hoffman, J.E. Spectroscopic scanning tunneling microscopy insights into Fe-based superconductors. Rep. Prog. Phys. 2011, 74, 124513. [CrossRef]

20. Cordin, M.; Amann, P.; Menzel, A.; Bertel, E.; Baranov, M.; Diehl, S.; Redinger, J.; Franchini, C. Comment on "Cleavage surfaces of the $\mathrm{BaFe}_{2-x} \mathrm{Co}_{x} \mathrm{As}_{2}$ and $\mathrm{Fe}_{y} \mathrm{Se}_{1-x} \mathrm{Te}_{x}$ superconductors: A combined STM plus LEED study". Phys. Rev. B 2012, 86, 167401. [CrossRef]

21. Gao, M.; Ma, F.; Lu, Z.-Y.; Xiang, T. Surface structures of ternary iron arsenides $A \mathrm{Fe}_{2} \mathrm{As}_{2}(A=\mathrm{Ba}, \mathrm{Sr}$, or $\mathrm{Ca})$. Phys. Rev. B 2010, 81, 193409. [CrossRef]

22. Gu, J.; Sim, W.S.; King, D.A. Vibrational-entropy-driven order-order phase transition at monolayer coverage: CO on Co $\{10-10\}$. J. Chem. Phys. 1997, 107, 5613-5616. [CrossRef]

23. Cordin, M.; Lechner, B.A.J.; Amann, P.; Menzel, A.; Bertel, E.; Franchini, C.; Zucca, R.; Redinger, J.; Baranov, M.; Diehl, S. Phase transitions driven by competing interactions in low-dimensional systems. EPL 2010, 92, 26004. [CrossRef]

24. Bertel, E. Quasi-critical fluctuations: A novel state of matter? J. Nanopart. Res. 2013, 15, 1-9. [CrossRef] [PubMed]

25. Deisl, C.; Swamy, K.; Memmel, N.; Bertel, E.; Franchini, C.; Schneider, G.; Redinger, J.; Walter, S.; Hammer, L.; Heinz, K. $(3 \times 1)-\mathrm{Br} / \mathrm{Pt}(110)$ structure and the charge-density-wave-assisted $\mathrm{c}(2 \times 2)$ to $(3 \times 1)$ phase transition. Phys. Rev. B 2004, 69, 195405-195413. [CrossRef]

26. Rice, T.M.; Scott, G.K. New mechanism for a charge-density-wave instability. Phys. Rev. Lett. 1975, 35, 120. [CrossRef]

27. Amann, P.; Cordin, M.; Braun, C.; Lechner, B.A.J.; Menzel, A.; Bertel, E.; Franchini, C.; Zucca, R.; Redinger, J.; Baranov, M.; et al. Electronically driven phase transitions in a quasi-one-dimensional adsorbate system. Eur. Phys. J. B 2010, 75, 15-22. [CrossRef]

28. Minca, M.; Penner, S.; Dona, E.; Menzel, A.; Bertel, E.; Brouet, V.; Redinger, J. Surface resonances on transition metals as low-dimensional model systems. N. J. Phys. 2007, 9, 386-399. [CrossRef]

29. Cordin, M.; Lechner, B.A.J.; Duerrbeck, S.; Menzel, A.; Bertel, E.; Redinger, J.; Franchini, C. Experimental observation of defect pair separation triggering phase transitions. Sci. Rep. 2014, 4, 4110. [CrossRef] [PubMed]

30. Zabolotnyy, V.B.; Evtushinsky, D.V.; Kordyuk, A.A.; Inosov, D.S.; Koitzsch, A.; Boris, A.V.; Sun, G.L.; Lin, C.T.; Knupfer, M.; Büchner, B.; et al. Fermi surface of $\mathrm{Ba}_{1-x} \mathrm{~K}_{x} \mathrm{Fe}_{2} \mathrm{As}_{2}$ as probed by angle-resolved photoemission. Phys. C Superconduct. 2009, 469, 448-451. [CrossRef]

31. Borisenko, S.V.; Zabolotnyy, V.B.; Kordyuk, A.A.; Evtushinsky, D.V.; Kim, T.K.; Morozov, I.V.; Follath, R.; Büchner, B. One-sign order parameter in iron based superconductor. Symmetry 2012, 4, 251-264. [CrossRef] 
32. Kordyuk, A.A.; Zabolotnyy, V.B.; Evtushinsky, D.V.; Yaresko, A.N.; Büchner, B.; Borisenko, S.V. Electronic band structure of ferro-pnictide superconductors from ARPES experiment. J. Superconduct. Novel Magnet. 2013, 26, 2837-2841. [CrossRef]

33. Kondo, T.; Fernandes, R.M.; Khasanov, R.; Liu, C.; Palczewski, A.D.; Ni, N.; Shi, M.; Bostwick, A.; Rotenberg, E.; Schmalian, J.; et al. Unexpected fermi-surface nesting in the pnictide parent compounds $\mathrm{BaFe}_{2} \mathrm{As}_{2}$ and $\mathrm{CaFe}_{2} \mathrm{As}_{2}$ revealed by angle-resolved photoemission spectroscopy. Phys. Rev. B 2010, 81, 060507. [CrossRef]

34. De Jong, S.; van Heumen, E.; Thirupathaiah, S.; Huisman, R.; Massee, F.; Goedkoop, J.B.; Ovsyannikov, R.; Fink, J.; Dürr, H.A.; Gloskovskii, A.; et al. Droplet-like fermi surfaces in the anti-ferromagnetic phase of $\mathrm{EuFe}_{2} \mathrm{As}_{2}$, an Fe-pnictide superconductor parent compound. EPL 2010, 89, 27007. [CrossRef]

35. Zhou, X.; Liu, G.; Meng, J.; Zhang, W.; Liu, H.; Zhao, L.; Jia, X. High resolution angle-resolved photoemission spectroscopy on Cu-based and Fe-based high-Tc superconductors. Phys. Status Solidi A 2010, 207, 2674-2692. [CrossRef]

36. Evtushinsky, D.V.; Kordyuk, A.A.; Zabolotnyy, V.B.; Inosov, D.S.; Kim, T.K.; Büchner, B.; Luo, H.; Wang, Z.; Wen, H.-H.; Sun, G.; et al. Propeller-like low temperature fermi surface of $\mathrm{Ba}_{1-x} \mathrm{~K}_{x} \mathrm{Fe}_{2} \mathrm{As}_{2}$ from magnetotransport and photoemission measurements. J. Phys. Soc. Jpn. 2011, 80, 023710. [CrossRef]

37. Zabolotnyy, V.B.; Inosov, D.S.; Evtushinsky, D.V.; Koitzsch, A.; Kordyuk, A.A.; Sun, G.L.; Park, J.T.; Haug, D.; Hinkov, V.; Boris, A.V.; et al. $(\pi, \pi)$ electronic order in iron arsenide superconductors. Nature 2009, 457, 569-572. [CrossRef] [PubMed]

38. Fink, J.; Charnukha, A.; Rienks, E.D.L.; Liu, Z.H.; Thirupathaiah, S.; Avigo, I.; Roth, F.; Jeevan, H.S.; Gegenwart, P.; Roslova, M.; et al. Non-fermi-liquid scattering rates and anomalous band dispersion in ferropnictides. Phys. Rev. B 2015, 92, 201106. [CrossRef]

39. Johannes, M.D.; Mazin, I.I. Fermi surface nesting and the origin of charge density waves in metals. Phys. Rev. B 2008, 77, 165135. [CrossRef]

40. Faraggi, M.N.; Zubizarreta, X.; Arnau, A.; Silkin, V.M. On the stability of the electronic system in transition metal dichalcogenides. J. Phys. Condens. Matter 2016, 28, 184004. [CrossRef] [PubMed]

41. Mazin, I.I.; Kimber, S.A.J.; Argyriou, D.N. Quasiparticle interference in antiferromagnetic parent compounds of iron-based superconductors. Phys. Rev. B 2011, 83, 052501. [CrossRef]

42. Song, C.-L.; Zhang, H.-M.; Zhong, Y.; Hu, X.-P.; Ji, S.-H.; Wang, L.; He, K.; Ma, X.-C.; Xue, Q.-K. Observation of double-dome superconductivity in potassium-doped FeSe thin films. Phys. Rev. Lett. 2016, 116, 157001. [CrossRef] [PubMed]

43. Ye, Z.R.; Zhang, C.F.; Ning, H.L.; Li, W.; Chen, L.; Jia, T.; Hashimoto, M.; Lu, D.H.; Shen, Z.-X.; Zhang, Y. Simultaneous emergence of superconductivity, inter-pocket scattering and nematic fluctuation in potassium-coated FeSe superconductor. Available online: http:/ /arxiv.org/abs/1512.02526 (acessed on 6 June 2016).

44. Blanco-Canosa, S.; Frano, A.; Loew, T.; Lu, Y.; Porras, J.; Ghiringhelli, G.; Minola, M.; Mazzoli, C.; Braicovich, L.; Schierle, E.; et al. Momentum-dependent charge correlations in $\mathrm{YBa}_{2} \mathrm{Cu}_{3} \mathrm{O}_{6+d}$ superconductors probed by resonant X-ray scattering: Evidence for three competing phases. Phys. Rev. Lett. 2013, 110, 187001. [CrossRef] [PubMed]

45. Da Silva Neto, E.H.; Aynajian, P.; Frano, A.; Comin, R.; Schierle, E.; Weschke, E.; Gyenis, A.; Wen, J.; Schneeloch, J.; Xu, Z.; et al. Ubiquitous interplay between charge ordering and high-temperature superconductivity in cuprates. Science 2014, 343, 393-396. [CrossRef] [PubMed]

46. Atkinson, W.A.; Kampf, A.P.; Bulut, S. Charge order in the pseudogap phase of cuprate superconductors. N. J. Phys. 2015, 17, 013025. [CrossRef]

47. Ghiringhelli, G.; Le Tacon, M.; Minola, M.; Blanco-Canosa, S.; Mazzoli, C.; Brookes, N.B.; De Luca, G.M.; Frano, A.; Hawthorn, D.G.; He, F.; et al. Long-range incommensurate charge fluctuations in $(\mathrm{Y}, \mathrm{Nd}) \mathrm{Ba}_{2} \mathrm{Cu}_{3} \mathrm{O}_{6+x}$. Science 2012, 337, 821-825. [CrossRef] [PubMed]

48. Comin, R.; Frano, A.; Yee, M.M.; Yoshida, Y.; Eisaki, H.; Schierle, E.; Weschke, E.; Sutarto, R.; He, F.; Soumyanarayanan, A.; et al. Charge order driven by fermi-arc instability in $\mathrm{Bi}_{2} \mathrm{Sr}_{2-x} \mathrm{La}_{x} \mathrm{CuO}_{6+\delta}$. Science 2014, 343, 390-392. [CrossRef] [PubMed]

49. Comin, R.; Sutarto, R.; da Silva Neto, E.H.; Chauviere, L.; Liang, R.; Hardy, W.N.; Bonn, D.A.; He, F.; Sawatzky, G.A.; Damascelli, A. Superconductivity. Broken translational and rotational symmetry via charge stripe order in underdoped $\mathrm{YBa}_{2} \mathrm{Cu}_{3} \mathrm{O}_{6+y}$. Science 2015, 347, 1335-1339. [CrossRef] [PubMed] 
50. Da Silva Neto, E.H.; Comin, R.; He, F.; Sutarto, R.; Jiang, Y.; Greene, R.L.; Sawatzky, G.A.; Damascelli, A. Charge ordering in the electron-doped superconductor $\mathrm{Nd}_{2-x} \mathrm{Ce}_{x} \mathrm{CuO}_{4}$. Science 2015, 347, $282-285$. [CrossRef] [PubMed]

51. Blackburn, E.; Chang, J.; Hücker, M.; Holmes, A.T.; Christensen, N.B.; Liang, R.; Bonn, D.A.; Hardy, W.N.; Rütt, U.; Gutowski, O.; et al. X-ray diffraction observations of a charge-density-wave order in superconducting ortho-II $\mathrm{YBa}_{2} \mathrm{Cu}_{3} \mathrm{O}_{6.54}$ single crystals in zero magnetic field. Phys. Rev. Lett. 2013, 110, 137004. [CrossRef] [PubMed]

52. Kohsaka, Y.; Taylor, C.; Fujita, K.; Schmidt, A.; Lupien, C.; Hanaguri, T.; Azuma, M.; Takano, M.; Eisaki, H.; Takagi, H.; et al. An intrinsic bond-centered electronic glass with unidirectional domains in underdoped cuprates. Science 2007, 315, 1380-1385. [CrossRef] [PubMed]

53. Wu, T.; Mayaffre, H.; Krämer, S.; Horvatić, M.; Berthier, C.; Hardy, W.N.; Liang, R.; Bonn, D.A.; Julien, M.-H. Incipient charge order observed by NMR in the normal state of $\mathrm{YbBa}_{2} \mathrm{Cu}_{3} \mathrm{O}_{y}$. Nat. Commun. 2015, 6, 6438. [CrossRef] [PubMed]

54. Wu, H.H.; Buchholz, M.; Trabant, C.; Chang, C.F.; Komarek, A.C.; Heigl, F.; Zimmermann, M.V.; Cwik, M.; Nakamura, F.; Braden, M.; et al. Charge stripe order near the surface of 12-percent doped $\mathrm{La}_{2-x} \mathrm{Sr}_{x} \mathrm{CuO}_{4}$. Nat. Commun. 2012, 3, 1023. [CrossRef] [PubMed]

55. Fine, B.V. Comment on "broken translational and rotational symmetry via charge stripe order in underdoped $\mathrm{YBa}_{2} \mathrm{Cu}_{3} \mathrm{O}_{6+y}$ ". Science 2016, 351, 235. [CrossRef] [PubMed]

56. Comin, R.; Sutarto, R.; Neto, E.H.D.S.; Chauviere, L.; Liang, R.; Hardy, W.N.; Bonn, D.A.; He, F.; Sawatzky, G.A.; Damascelli, A. Response to comment on "Broken translational and rotational symmetry via charge stripe order in underdoped $\mathrm{YBa}_{2} \mathrm{Cu}_{3} \mathrm{O}_{6+y}$ ”. Science 2016, 351, 235. [CrossRef] [PubMed]

57. Wang, Y.; Chubukov, A.V. Enhancement of superconductivity at the onset of charge-density-wave order in a metal. Phys. Rev. B 2015, 92, 125108. [CrossRef]

58. Soshi, I.; Motoharu, I. Possibility of charge density wave transition in a $\mathrm{SrPt}_{2} \mathrm{Sb}_{2}$ superconductor. J. Phys. Condens. Matter 2016, 28, 165702.

59. Kiss, T.; Yokoya, T.; Chainani, A.; Shin, S.; Hanaguri, T.; Nohara, M.; Takagi, H. Charge-order-maximized momentum-dependent superconductivity. Nat. Phys. 2007, 3, 720-725. [CrossRef]

60. Dressel, M. Quantum criticality in organic conductors? Fermi liquid versus non-fermi-liquid behaviour. J. Phys. Condens. Matter 2011, 23, 293201. [CrossRef] [PubMed]

61. Wen, C.H.P.; Xu, H.C.; Chen, C.; Huang, Z.C.; Lou, X.; Pu, Y.J.; Song, Q.; Xie, B.P.; Abdel-Hafiez, M.; Chareev, D.A.; et al. Anomalous correlation effects and unique phase diagram of electron-doped FeSe revealed by photoemission spectroscopy. Nat. Commun. 2016, 7, 10840. [CrossRef] [PubMed]

(C) 2016 by the authors; licensee MDPI, Basel, Switzerland. This article is an open access article distributed under the terms and conditions of the Creative Commons Attribution (CC-BY) license (http://creativecommons.org/licenses/by/4.0/). 